\section{West Nile virus encephalitis induced opsoclonus-myoclonus syndrome}

\author{
Chad J. Cooper, Sarmad Said \\ Department of Internal Medicine, Texas \\ Tech University Health Sciences Center, \\ EI Paso, TX, USA
}

\section{Abstract}

West Nile virus (WNV) is an arthropod borne neurotropic single stranded RNA flavivirus with $<1 \%$ developing presenting with neurological disease. Immunocompromised and elderly patients are more prone to developing WNV meningitis or encephalitis. Definitive diagnosis of WNV meningoencephalitis is a combination of clinical suspicion and cerebrospinal fluid (CSF) serology. Forty-eight year old Caucasian female presented with a sudden onset of altered mental status after being found unresponsive. She was confused with intermittent bouts of alertness/lethargy and unintelligible responses to questioning. Her medical problems included endometrial cancer that was in remission after undergoing a total abdominal hysterectomy with bilateral salpingectomy and postoperative chemotherapy with paclitaxel and carboplatin. Pertinent physical examination revealed muscle strength that was significantly decreased, nuchal rigidity and +2 pitting edema of both lower extremities. Computed tomography and magnetic resonance imaging of the brain were negative for any intracranial pathology. CSF analysis was consistent with aseptic meningitis with all CSF serology being negative except for positive WNV antibody. A few days after being admitted she developed involuntary random movements of her eyes and generalized jerking movements (myoclonus). This was determined to be opsoclonus myoclonus syndrome (OMS) induced by the WNV meningoencephalitis. She then received five consecutive days of plasmapheresis with a significant improvement in her neurological status. Opsoclonus-myoclonus syndrome (OMS) is a rare neurological disorder associated with chaotic multidirectional eye movements, myoclonus and less frequently cerebellar ataxia. OMS affects as few as 1 in $10,000,000$ people per year. The pathogenesis is not fully understood with the majority of cases of opsoclonus-myoclonus syndrome being idiopathic. According to current medical literature there have only been two previous case reports of opsoclonus myoclonus syndrome associated with WNV encephalitis.

\section{Introduction}

West Nile virus (WNV) is an arthropod borne neurotropic single stranded RNA flavivirus. ${ }^{1}$ The virus is introduced into the host by the vector (Culex mosquito) during its blood meal. WNV originated in the Middle East and Africa but in recent years has reached the United States.2 From 1999 to 2011, a total of 31,414 cases of WNV were reported in the United States. The 2012 incidence of WNV neuroinvasive disease was 0.3 per 100,000 in the United States. ${ }^{3}$ Approximately $45 \%$ of WNV cases were reported in Texas, USA. ${ }^{3}$ Seasonal outbreaks occur annually but are often quite focal and unpredictable in size and location.

The majority of those infected with WNV are asymptomatic and only $20-30 \%$ will present with flu like symptoms. Less than $1 \%$ of individuals will eventually develop neurological deficits. Neurological deficits associated with WNV infection can be encephalitis, meningitis or flaccid paralysis. Immunocompromised and elderly patients are more prone to developing WNV meningitis or encephalitis compared to the general population. ${ }^{4} \mathrm{WNV}$ and other arboviral infections should be considered in the differential diagnosis of aseptic meningitis or encephalitis, especially if the geographical area is prone to WNV infection. The definitive diagnosis of WNV meningoencephalitis is a combination of clinical suspicion and CSF serology. Approximately 50\% may have significant postviral morbidity for a year following infection. ${ }^{4}$ Management of WNV infection consists of supportive measures. We are presenting a very rare case of a immunocompromised patient that developed opsoclonus myoclonus syndrome following WNV encephalitis.

\section{Case Report}

Forty-eight year old Caucasian female presented with a sudden onset of altered mental status after being found unresponsive. She was confused with intermittent bouts of alertness/lethargy and unintelligible responses to questioning. Her medical problems included endometrial cancer that was treated with a total abdominal hysterectomy with bilateral salpingectomy and postoperative chemo therapy. The endometrial adenocarcinoma was a stage 2 according to the International Federation of Gynecology and Obstetrics (FIG0) staging: $8 \mathrm{~cm}$ tumor, grade 2, no lymph node involvement, and $<50 \%$ invasion of the myometrium and cervical stroma. The patient was currently in remission after receiving chemotherapy (paclitaxel and carboplatin) and dexamethasone. No history of tobacco, alcohol or illicit drug use.
Correspondence: Chad J. Cooper, Department of Internal Medicine, Texas Tech University Health Sciences Center, 4800 Alberta Ave, El Paso, TX 79905, USA

Tel. +1.915.543.1009.

E-mail: chad.cooper@ttuhsc.edu

Key words: West Nile virus, opsoclonus myoclonus syndrome, encephalitis.

Contributions: the authors contributed equally.

Conflict of interests: the authors declare no potential conflict of interests.

Received for publication: 18 February 2014. Accepted for publication: 19 February 2014.

This work is licensed under a Creative Commons Attribution NonCommercial 3.0 License (CC BYNC 3.0).

(C) Copyright C.J. Cooper and S. Said, 2014

Licensee PAGEPress, Italy

Neurology International 2014; 6:5359

doi:10.4081/ni.2014.5359

Initial vital signs on admission included a rectal temperature was 41.1 degrees Celsius, tachycardia (heart rate of 108), stage 1 hypertension (blood pressure of 140/61) and tachypnea (respiratory rate of 34 ). Pertinent physical examination findings revealed a morbidly obese female that was alert but not oriented to person, place or time and only able to answer yes or no to questioning. Her pupils were $3 \mathrm{~mm}$ bilaterally, equal round and reactive to light with intact extraocular movements and no nystagmus noted. All cranial nerves were assessed and normal on examination. No motor or sensory deficits to light, touch, pain, position sense or vibration noted. All reflexes such as biceps, knee tendon and Babinski sign were normal. Muscle strength ( $3 / 5$ all four extremities) but no tremors, or myoclonus. Nuchal rigidity was observed but Kernig and Brudzinski signs were both negative. She also had bilateral +2 pitting edema of lower extremities. The remainder of organ systems of examination was unremarkable.

Initial laboratory work-up (Table 1) was unremarkable except hyponatremia and thrombocytopenia. Brain computed tomography (CT) on admission was negative for any acute pathological abnormalities. CT of the abdomen and pelvis was negative for any acute abdominopelvic pathology. The patient was started on ceftriaxone $2 \mathrm{~g}$ IV daily, vancomycin $2 \mathrm{~g}$ IV twice daily, and acyclovir $1.5 \mathrm{~g}$ IV every 8 hours due to our high suspicion of encephalitis. A lumbar puncture with cerebrospinal fluid (CSF) analysis and CSF serology (Table 2) was consistent with aseptic meningitis. The HIV screening test was negative. Magnetic reso- 
nance imaging (MRI) of the brain was unremarkable for any acute intracranial abnormalities. Vancomycin and acyclovir were discontinued and fluconazole $400 \mathrm{mg}$ PO daily was added to the management on the sixth hospital and ceftriaxone was continued until the tenth hospital day. By the seventh hospital day, we noted involuntary random movements of her eyes (see Video 1) and generalized jerking movements (myoclonus), mostly in lower extremities. An electroencephalography (EEG) showed a severe and diffuse slowness, consistent with encephalopathy. She was placed on fosphenytoin $100 \mathrm{mg}$ IV every 8 hours without any improvement. On the eighth hospital day, her GCS decreased to 3 and was subsequently intubated. A repeat brain CT did not reveal any new pathologic abnormalities. The platelet count continued to decrease during the hospitalization and eventually reached its lowest count of $21 \times 10^{3 / u L}$ by the tenth hospital day. The West Nile virus (WNV) antibody IgM and IgG were noted to be positive on the twelfth hospital day. After determining that the encephalitis was due to the WNV we continued supportive care and discontinued the fluconazole. The neurology service was consulted for the myoclonus and erratic eye movements. They diagnosed her to have opsoclonus myoclonus syndrome (OMS) induced by the WNV meningoencephalitis. Paraneoplastic antibodies anti-Yo, anti-Hu, anti-Ri and antiNMDAR were negative. Neurology recommended a trial of either plasmapheresis or IVIg. Therefore the patient had plasmapheresis on five consecutive days. A percutaneous endoscopic gastrostomy tube (PEG) and tracheostomy were performed on hospital day 26 . The patient continued to have improvement in her neurological status and was subsequently discharge to a long term acute care (LTAC) facility for further rehabilitation. She underwent rehabilitation for six months and eventually had complete resolution of her neurological deficits on follow visit.

\section{Discussion and Conclusions}

Opsoclonus-myoclonus syndrome (OMS) is a rare neurological disorder associated with chaotic multidirectional eye movements, myoclonus and less frequently cerebellar ataxia. OMS affects as few as 1 in 10,000,000 people per year. 5 The eye movements are characterized by multidirectional, high amplitude, arrhythmic and conjugate ocular saccadic intrusions without intersaccadic latency causing oscillopsia. ${ }^{6}$ It should not be confused with nystagmus which is usually unidirectional and has a slow component. ${ }^{7}$ Myoclonus may affect any part of the body and may be evoked by action, intention, auditory and visual stimuli.
The onset of myoclonus may precede the opsoclonus.

The pathogenesis of opsoclonus is unknown but, one hypothesis suggests that opsoclonus is due to damage of the omnipause cells in the nucleus raphe interpositus of the pons. ${ }^{8}$ This prevents unwanted saccades by inhibiting burst neurons in the paramedian pontine reticular formation and rostral interstitial nucleus of Cajal. However, these omnipause neurons are spared in paraneoplastic opsoclonus. Another explanation suggests that opsoclonus may be generated by disinhibition of the oculomotor region of the fastigial nucleus by a process that interferes with the inhibitory projections of the Purkinje cells of the deep cerebellar nuclei to the fastigial nuclei. 8 The pathogenesis is not fully understood, however, an autoimmune pathophysiology has been proposed. Autoimmune dysfunction of Purkinje cells in the dorsal vermis and subsequent disinhibition of oculomotor fastigial region seems to be the most probable mechanism..$^{9}$ The majority of OMS cases are idiopathic. However, other common causes of opsoclonus include paraneoplastic, viral brainstem encephalitis and drugs. ${ }^{10,11}$ The onconeuronal antibodies associated with OMS are anti$\mathrm{Ri}$ antibodies (gynecologic cancers) and, less frequently, anti-Hu, anti-Yo, and anti-Ma-2

Table 1. Initial laboratory work up.

\begin{tabular}{lc}
\hline White blood cell count & $8.09 \times 10^{3}$ uL $\left(4.5-11.0 \times 10^{3} / \mathrm{UL}\right)$ \\
Segmenters & $53.1 \%$ \\
Bands & $11.3 \%$ \\
\hline Metamyelocytes & $1 \%$ \\
Lymphocytes & $24.4 \%$ \\
\hline Monocytes & $10.4 \%$ \\
Hemoglobin & $39.2 \mathrm{~g} / \mathrm{dL}(12.0-15.0 \mathrm{~g} / \mathrm{dL})$ \\
\hline Hematocrit & $109 \times 10^{3} \mathrm{uL}(150-450 \times 103 / \mathrm{dL})$ \\
Platelet count & $133 \mathrm{mmol} / \mathrm{L}(135-145 \mathrm{mmol} / \mathrm{L})$ \\
\hline Sodium & $4.4 \mathrm{mmol} / \mathrm{L}(3.5-5.1 \mathrm{mmol} / \mathrm{L})$ \\
Potassium & $119 \mathrm{mg} / \mathrm{dL}(70-100 \mathrm{mg} / \mathrm{dL})$ \\
\hline Serum glucose & $21 \mathrm{mg} / \mathrm{dL}(7-22 \mathrm{mg} / \mathrm{dL})$ \\
BUN & $1.50 \mathrm{mg} / \mathrm{dL}(0.60-1.30 \mathrm{mg} / \mathrm{dL})$ \\
\hline Creatinine & $20 \mathrm{IU} / \mathrm{L}(15-37 \mathrm{IU} / \mathrm{L})$ \\
AST & $36 \mathrm{IU} / \mathrm{L}(12-78 \mathrm{IU} / \mathrm{L})$ \\
\hline ALT & $128 \mathrm{IU} / \mathrm{L}(50-136 \mathrm{umol} / \mathrm{L})$ \\
Alk. Phosphatase &
\end{tabular}

Table 2. Cerebrospinal fluid analysis.

\begin{tabular}{lc} 
Appearance & Clear and colorless \\
White blood cell count & $67 / \mathrm{uL}(0-5 / \mathrm{uL})$ \\
Neutrophils & $1 \%$ \\
\hline Lymphocytes & $97 \%$ \\
Monocytes & $2 \%$ \\
\hline Red blood cell count & $399 / \mathrm{uL}(0-5 / \mathrm{uL})$ \\
Protein & $131 \mathrm{mg} / \mathrm{dL}(15-45 \mathrm{mg} / \mathrm{dL})$ \\
\hline Glucose & $38 \mathrm{mg} / \mathrm{dL}(41-70 \mathrm{mg} / \mathrm{dL})$ \\
Corresponding serum glucose & $68 \mathrm{mg} / \mathrm{dL}(70-100 \mathrm{mg} / \mathrm{dL})$ \\
\hline CSF serology & \\
Cryptococcal antigen & Negative \\
Cysticerosis IgG antibody & Negative \\
Coccidioides antibody & Negative \\
Histoplasma antibody & Negative \\
Group B Streptococci & Negative \\
Haemophilus & Negative \\
Streptococcus pneumonia & Negative \\
Neisseria meningitides & Negative \\
Herpes simplex 1 \& 2Negative & Positive \\
West Nile Virus & Negative \\
CSF Culture & Negative \\
CSF cytology & No malignancy \\
\hline
\end{tabular}


antibodies.12 Paraneoplastic diseases associated with OMS include small-cell lung, ovarian and breast cancer. ${ }^{13}$ Most infectious disease causes involve the central nervous system or a post infection immune mediated process. The post infectious mechanism has been described as a dysfunction of the cell mediated and humoral immune response. This immune mediated process was postulated because the presence of autoantibodies and the response of OMS to immunosuppressive therapy. ${ }^{14}$ These autoantibodies are directed towards certain neural antigens. The specific microorganism is rarely identified however OMS has been reported to occur after infection with enterovirus, Borrelia Burgdorferi, streptococcus, Coxsackie virus, Epstein-Barr virus, mumps or West Nile virus. 15

Opsoclonus myoclonus syndrome can result in significant long term neurological sequelae if either untreated or undertreated.16 There is no current established treatment for OMS. Therapy options have been directed towards the suppression of antibody formation and the removal of the autoantibodies. Several therapeutic attempts can be utilized to remove the autoantibodies in patients with OMS, such as IVIG, plasmapheresis, and immunoadsorption. ${ }^{16}$ Treatment of the underlying disease process is the mainstay of management for OMS. However there is no available data from clinical trials that suggest a definitive treatment strategy. Because the opsoclonusmyoclonus syndrome is so rare, there are no randomized controlled trials to provide guide- lines for either symptomatic or disease modifying treatments. According to current medical literature, only two other case reports have reported opsoclonus myoclonus syndrome induced by West Nile virus encephalitis.

\section{References}

1. Steiner I, Kennedy PG. West Nile virus introduction into the new world. Neurology 2013;81:1441-2.

2. Rossini G, Landini MP, Gelsomno F, et al. Innate host responses to West Nile virus: implications for central nervous system immunopathology. World J Virol 2013;2:4956.

3. Centers for Disease Control and Prevention. West Nile Virus. 2013. Available from: www.cdc.gov/westnile/

4. Morita A, Ishihara M, Kamei S, Ishikawa H. Opsoclonus-myoclonus syndrome following influenza an infection. Intern Med 2012;51:2429-31.

5. Singh D, Sinha M, Kumar R, et al. Opsoclonus-myoclonus syndrome caused by varicella-zoster virus. Ann Indian Acad Neurol 2010;13:211-2.

6. Guedes ME, Almeida AC, Patricio MS, Costa JM. Opsoclonus-myoclonus syndrome. BMJ Case Rep 2011;28:2011.

7. Sahu JK, Prasad K. The opsoclonusmyoclonus syndrome. Pract Neurol 2011; 11:160-6.
8. Wong A. An update on opsoclonus. Curr Opin Neurol 2007;20;25-31.

9. Morales-Briceno H, Rodriguez-Violante M, Cervantes-Arriage A, et al. Opsoclonus myoclonus syndrome associated with GQ1b antibodies. Mov Disorders 2012;27: 1615-6.

10. Scott KM, Parker F, Heckmann JM. Opsoclonus-myoclonus syndrome and HIVinfection. J Neurol Sci 2009;284:192-5.

11. Khosla JS, Edelman MJ, Kennedy N, Reich SG. West Nile virus presenting as opsoclonus-myoclonus cerebellar ataxia. Neurology 2005;64:1095.

12. Alshekhlee A, Sultan B, Chandar K. Opsoclonus persisting during sleep in West Nile encephalitis. Arch Neurol 2006; 63:1324-6.

13. Ertekin V, Tan H. Opsoclonus-myoclonus syndrome attributable to hepatitis $\mathrm{C}$ infection. Pediatr Neurol 2010;42:441-2.

14. Yiu VW, Kovithavongs T, McGonigle LF, Ferrerira P. Plasmapheresis as an effective treatment for opsoclonus-myoclonus syndrome. Pediatr Neurol 2001;24:72-4.

15. Kanjanasut N, Phanthumchinda K, Bhidayasiri R. HIV-related opsoclonusmyoclonus-ataxia syndrome: Report on two cases. Clin Neurol Neurosur 2010;112: $572-4$.

16. Desai J, Mitchell WG. Acute cerebellar ataxia, acute cerebellitis, and opsoclonusmyoclonus syndrome. J Child Neurol 2012; 27:1482-8. 